\title{
MODELADO DINÁMICO DE UN SISTEMA DE ALMACENAMIENTO DE FRÍO VINCULADO A UN CICLO DE REFRIGERACIÓN
}

\author{
Guillermo Bejarano, José Joaquín Suffo, Manuel Vargas, Manuel G. Ortega \\ Departamento de Ingeniería de Sistemas y Automática, Universidad de Sevilla \\ gbejarano@us.es, sufagui@gmail.com, \{mvargas, mortega\}@us.es
}

\begin{abstract}
Resumen
En este trabajo se estudia el modelado dinámico de un sistema de almacenamiento de frío basado en material de cambio de fase, que permita la gestión del frío generado por una planta experimental de refrigeración por compresión de vapor, basada en criterios económicos y de satisfacción de la demanda. Se describe el diseño del sistema de almacenamiento y se propone un modelo dinámico simplificado que permita estudiar la carga y descarga del mismo. Se incluyen algunas simulaciones de carga y descarga, realizadas considerando condiciones de contorno entálpicas y másicas del refrigerante y fluido secundario acordes a las generadas por la planta experimental.
\end{abstract}

Palabras clave: Sistemas de refrigeración, Almacenamiento de energía térmica, Materiales de cambio de fase, Modelado dinámico.

\section{INTRODUCCIÓN}

La demanda de energía requerida por los sistemas de refrigeración se ha convertido en un pilar esencial en la estructura energética. Esta demanda está creciendo significativamente en los últimos años, tanto en aplicaciones industriales y comerciales, como para sistemas de refrigeración doméstica [16]. Algunos informes elevan al $30 \%$ el total de la energía consumida en todo el mundo asociada a sistemas de acondicionamiento de aire o HVAC (del inglés Heating, Ventilating and Air Conditioning) [4, 9], mientras que el informe más reciente sobre consumo de energía doméstica en EEUU señala que el $28 \%$ de la energía consumida en hogares norteamericanos se debe a refrigeradores y sistemas HVAC [24].

La generación de frío mediante sistemas de compresión de vapor, véase la Figura 1, es sin duda el método más utilizado a nivel mundial. Se utiliza un fluido refrigerante para extraer calor del flujo secundario de un evaporador a baja presión, de manera que dicho calor es cedido al ambiente mediante un condensador que trabaja a presión más alta. El compresor se encarga de elevar la presión del refrigerante, constituyendo el principal aporte energético externo para el funcionamiento del ciclo.

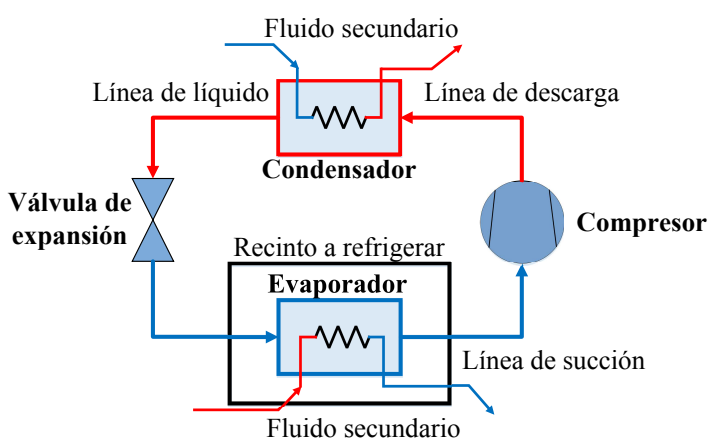

Figura 1: Sistema de refrigeración por compresión de vapor

Este método ha venido siendo utilizado desde hace décadas, si bien en sus orígenes el rendimiento energético dejaba mucho que desear. La aparición en los últimos años de nuevos diseños de sus elementos, así como el hecho de que el control automático ha permitido la incorporación de algoritmos que adaptan de forma continua los parámetros del sistema a las condiciones de trabajo, tanto en estado estacionario como en transitorio, ha permitido mejorar sensiblemente el Coeficiente de Comportamiento ( $C O P$, del inglés Coefficient of Performance). A pesar de ello, y debido a la creciente escasez de fuentes energéticas, el interés en seguir mejorando la eficiencia energética y reducir el impacto ambiental de los sistemas actuales se ha visto incrementado en los últimos años.

Recientemente están apareciendo trabajos enfocados no solo a la generación de frío, sino también a la gestión del mismo. Una línea muy destacada es la incorporación de sistemas de almacenamiento de energía (TES, del inglés Thermal Energy Storage) para frío, de manera que se utilicen como reservorios donde almacenar excesos de energía frigorífica y extraerla cuando sea necesario. Entre las ventajas ofrecidas por estos sistemas de almacenamiento, destacan la oportunidad de flexibilizar las condiciones de operación, el diseño de equipos más 
Tabla 1: Nomenclatura

\begin{tabular}{|c|c|c|c|}
\hline \multicolumn{2}{|c|}{ Símbolos latinos } & \multicolumn{2}{|c|}{ Subíndices y superíndices } \\
\hline$C O P$ & Coeficiente de Comportamiento & cond & conducción \\
\hline$c_{p}$ & Calor específico a presión constante $\left[\mathrm{J} \mathrm{kg}^{-1} \mathrm{~K}^{-1}\right]$ & conv & convección \\
\hline$e$ & Espesor $[\mathrm{m}]$ & env & entorno \\
\hline$h$ & Entalpía específica $\left[\mathrm{J} \mathrm{kg}^{-1}\right]$ & ext & exterior \\
\hline$\dot{m}$ & Caudal másico $\left[\mathrm{kg} \mathrm{s}^{-1}\right]$ & $i n$ & entrada \\
\hline$n$ & Número de elementos & ints & interno \\
\hline$P C M$ & Material de cambio de fase & intm & fluido intermedio \\
\hline$\dot{Q}$ & Potencia térmica $[\mathrm{W}]$ & lat & estado latente \\
\hline$R$ & Resistencia térmica $\left[\mathrm{K} \mathrm{W}^{-1}\right]$ & lat- & entalpía mínima en zona latente \\
\hline$r$ & Radio interior $[\mathrm{m}]$ & lat+ & entalpía máxima en zona latente \\
\hline$T$ & Temperatura $[\mathrm{K}]$ & out & salida \\
\hline$T E S$ & Sistema de almacenamiento de energía & pcm & PCM \\
\hline$t$ & Tiempo $[\mathrm{s}]$ & $\mathrm{pcm}_{l}$ & PCM en fase líquida \\
\hline & Volumen $\left[\mathrm{m}^{3}\right]$ & $\mathrm{pcm}_{\mathrm{s}}$ & PCM en fase sólida \\
\hline \multicolumn{2}{|c|}{ Símbolos griegos } & ref & refrigerante \\
\hline$\alpha$ & $\begin{array}{l}\text { Coeficiente de transferencia de calor por } \\
\text { convección }\left[\mathrm{W} \mathrm{m} \mathrm{m}^{-2} \mathrm{~K}^{-1}\right]\end{array}$ & $r e f_{v}$ & refrigerante en fase vapor \\
\hline$\varepsilon$ & Eficiencia del intercambio de calor & $r e f_{2}$ & refrigerante en zona bifásica \\
\hline$\zeta$ & Fracción de la longitud total del intercambiador & $s e c$ & fluido secundario \\
\hline$\kappa$ & Conductividad térmica $\left[\mathrm{W} \mathrm{m}^{-1} \mathrm{~K}^{-1}\right]$ & wall & pared \\
\hline$\rho$ & Densidad $\left[\mathrm{kg} \mathrm{m}^{-3}\right]$ & & \\
\hline
\end{tabular}

pequeños, un uso más eficiente de los mismos, así como la reducción del consumo de energía [7]. De hecho, el hecho de disponer de un sistema auxiliar capaz de almacenar el exceso de frío evita que el equipo sea sobredimensionado para hacer frente a los períodos de alta demanda [10].

En el contexto de un sistema de refrigeración, la posibilidad de aprovechar el precio bajo de la energía eléctrica durante los períodos de menor demanda se valora positivamente. Sin embargo, la demanda de frío debe ser satisfecha en tiempo real, por tanto la idea de disponer de un almacén de frío que pueda ser usado en períodos de alta demanda puede evitar la necesidad de generar frío en dichos períodos, con el consiguiente ahorro económico (peak-shifting) [6, 18].

En el diseño de estos reservorios de frío, los sistemas basados en materiales de cambio de fase (PCM, del inglés Phase-Change Material) se han impuesto como la opción más atractiva por encima de los materiales de calor sensible, debido a sus buenas propiedades termodinámicas para el intercambio de calor, no solo por una adecuada capacidad calorífica, sino también porque la temperatura de dichos materiales no varía apreciablemente siempre que se encuentren en zona latente [11]. Esto permite almacenar la misma cantidad de energía en un volumen inferior y permite un intercambio de calor más eficiente, ya que la diferencia de temperatura entre la fuente de frío y el fluido receptor permanece uniforme a lo largo del intercambiador de calor. Por ejemplo, Oró et al. ofrece una clasificación de materiales actuales de cambio de fase sólido-líquido para aplicaciones de almacenamiento de frío, indicando temperatura de fusión y capacidad calorífica en la zona latente, además de otras propiedades físicas [14].

Pero si bien es importante el diseño del sistema, tanto desde el punto de vista de la elección del material como de su capacidad de almacenamiento, no lo es menos la gestión del mismo, a fin de optimizar índices de comportamiento. Una introducción sobre esta tecnología puede encontrarse en los trabajos publicados por Wang et al. [25, 26, 27]. En el primero de ellos se aborda el diseño de una planta de gran escala para un sistema de aire acondicionado apoyado por un anillo de TES basados en PCM [25]. En el segundo se muestra el modelado del sistema [26], mientras que en el tercero se propone una estrategia de control básica para la gestión del mismo, consistente en la activación de módulos situados en distintos emplazamientos de la instalación [27]. Asimismo, otros trabajos presentan un análisis exergético para medir el comportamiento de un sistema de aire acondicionado cuando se incorporan múltiples TES basados en PCM, tomando como estrategia de control el uso combinado de los distintos módulos $[12,13]$.

Trabajos más recientes utilizan control predictivo para la gestión de sistema de refrigeración. Por ejemplo, Shafiei et al. presenta un esquema de control MPC para minimizar las desviaciones de consumo de energía eléctrica respecto a un consumo de referencia en una planta de refrigeración de 
gran escala [21]. Para ello utiliza la energía cargada y descargada del PCM, introduciendo en el problema de optimización una referencia de la temperatura de evaporación como una variable manipulable ficticia. Posteriormente, Shafiei et al. presenta un controlador predictivo para la gestión del sistema de refrigeración de un camión con cámara frigorífica, al cual se le incorpora en paralelo un tanque con PCM [20]. Se utilizan el perfil de la ruta e información del tráfico, así como de las condiciones ambientales, para predecir la demanda de carga térmica durante períodos de tráfico denso. Por su parte, Schalbart et al. presenta la aplicación del control MPC a la gestión de un almacén frigorífico de helados conectado a un TES basado en PCM [19]. El controlador está basado en las curvas estáticas del ciclo de refrigeración y en el balance energético entre el ciclo y el tanque, tratando de garantizar la calidad del helado mientras intenta reducir el consumo de energía. En esta configuración el depósito PCM está en serie, de manera que un sistema de refrigeración de potencia variable carga al TES, y este a su vez es descargado para enfriar el almacén frigorífico.

En el presente trabajo se aborda el modelado dinámico de un tanque TES dedicado al almacenamiento de frío generado en una planta experimental de refrigeración por compresión de vapor, lo que determina las condiciones de contorno de los fluidos de carga y descarga. El diseño estructural del sistema de almacenamiento es propio, así como el enfoque de modelado, que es totalmente parametrizable y permite tanto la simulación dinámica de procesos de carga y descarga completos, como el diseño de los parámetros de la instalación (propiedades del PCM, número y diámetro de esferas de PCM, longitud y diámetro de tubos, etc). Así, en la Sección 2 se describe el tanque TES a modelar y su conexión con la planta experimental; en la Sección 3 se describen las suposiciones realizadas y se detalla el modelo dinámico del sistema, mientras que en la Sección 4 se analizan algunos resultados de simulación obtenidos para procesos de carga y descarga. Finalmente, en la Sección 5 se resumen las principales conclusiones y se proponen algunos trabajos futuros.

\section{DESCRIPCIÓN DEL SISTEMA}

La Figura 2(a) muestra un diagrama de la planta experimental a la que se pretenden incorporar bloques TES basados en PCM. Aunque se han incluido dos compresores y dos evaporadores con sus respectivas válvulas de expansión, la planta se puede configurar para operar con una o dos etapas de compresión y con uno o dos recintos.
Se dispone de variadores de frecuencia para los dos compresores y todas las bombas de líquido, para un control más preciso de su velocidad. Ambos evaporadores son intercambiadores de placas, conectados a dos bucles de fluido secundario (solución acuosa de glicol) controlados en flujo (por las bombas de líquido) y temperatura (mediante las resistencias eléctricas). El Evaporador 1 debe trabajar en torno a $5^{\circ} \mathrm{C}$ y el Evaporador 2 aproximadamente a $-20^{\circ} \mathrm{C}$, ya que son niveles de referencia para refrigeración. Existen dos posibilidades para el condensador: bien un intercambiador de flujo cruzado refrigerado por aire o bien un intercambiador de placas que utiliza agua como fluido secundario. Las válvulas de expansión electrónica se controlan mediante modulación por ancho de pulso (PWM), mientras que el refrigerante usado es R404a. La planta tiene instrumentación completa: se han instalado termopares y transductores de presión en todos los puntos relevantes del ciclo, para poder calcular las entalpías específicas, y se mide el caudal volumétrico de los fluidos secundarios mediante caudalímetros magnéticos de inducción. Se puede consultar más información sobre los componentes de la planta en [2].

Se pretende añadir a esta planta dos bloques TES basados en PCM en paralelo con los dos recintos a refrigerar, uno para el recinto a $5^{\circ} \mathrm{C}$ (TES1) y otro para el recinto a $-20^{\circ} \mathrm{C}$ (TES2), lo cual implicaría modificar la planta con dos líneas adicionales de refrigerante, con sus correspondientes válvulas de expansión, de manera que se habilite la posibilidad de cargar los TES. La descarga de los mismos se realizará mediante dos bombas que permitan recircular el fluido de los recintos a refrigerar por los TES correspondientes, tal como se muestra en la Figura 2(b).

En cuanto al diseño de los bloques TES, el PCM debe ser encapsulado de forma que las dos fases (sólido y líquido) permanezcan en contacto. Existen tres tipos de encapsulado [14, 17]: a granel, en el cual el PCM se almacena directamente dentro del tanque; el macroencapsulado, en el cual el PCM está formado por estructuras de diámetro superior a $1 \mathrm{~cm}$ recubiertas por una película polimérica, y por último el microencapsulado, donde las estructuras encapsuladas son aún más pequeñas e igualmente están recubiertas por un polímero.

En esta aplicación se ha seleccionado el macroencapsulado, de forma que el tanque de almacenamiento contiene esferas de PCM bañadas en un líquido de alta conductividad térmica y baja capacidad calorífica que constituye el lecho. En contacto con el mismo se instalan dos haces de tubos que atraviesan el tanque. El primero está destina- 


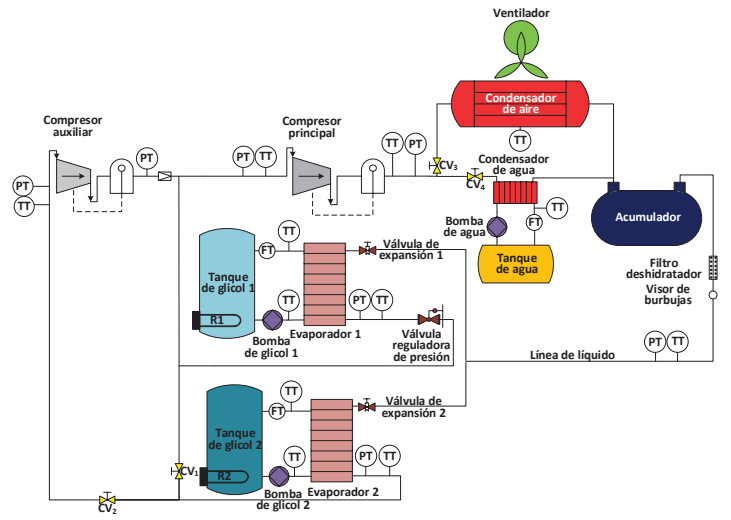

(a) Diagrama esquemático de la planta experimental

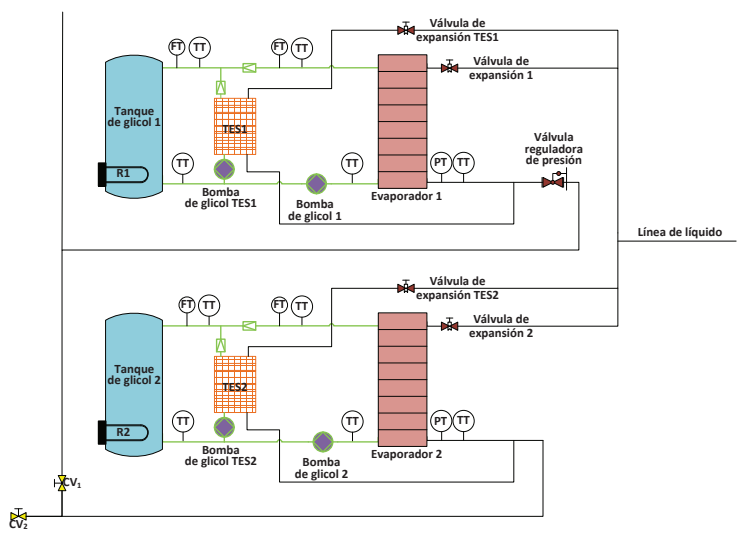

(b) Detalle de la incorporación de bloques TES a la planta experimental de refrigeración

Figura 2: Planta experimental de refrigeración

do a la circulación del refrigerante, por tanto el tanque actúa como un evaporador donde el refrigerante se evapora mientras extrae calor del lecho y por tanto de las esferas de PCM. Por su parte, la solución acuosa de glicol circula por el segundo haz de tubos, cediendo calor al lecho y por tanto a las esferas de PCM. Esta estructura es similar a la propuesta por Tay et al. [23], mientras que el lecho está basado en la tecnología STL desarrollada por CRISTOPIA Energy Systems [5]. El esquema propuesto para el tanque TES se muestra en la Figura 3.

\section{MODELADO DINÁMICO}

Para el modelado dinámico del tanque TES se realizan algunas simplificaciones:

(a) Se considera que el fluido intermedio que baña los tubos y las esferas de PCM tiene la misma temperatura en cualquier punto del tanque, debido a su alta conductividad térmica [1]. Esto simplifica el modelado ya que se trata de un modelo de parámetros concentrados

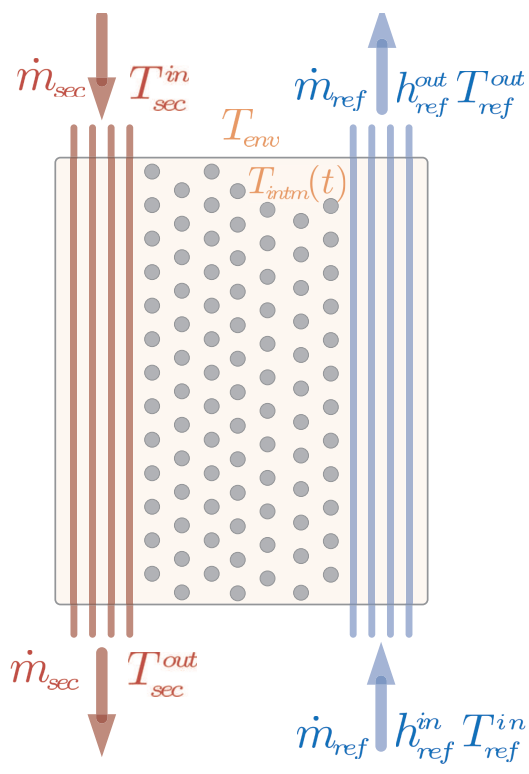

Figura 3: Esquema del tanque TES

y por tanto no se considera variación espacial del lado del fluido intermedio. Por tanto, este actúa simplemente como un integrador, interactuando con las potencias térmicas intercambiadas con las esferas de PCM, los tubos y el ambiente.

(b) El superenfriamiento es un fenómeno bien conocido en el ámbito de PCM [22]. Este fenómeno consiste en que es necesario bajar la temperatura por debajo del punto de congelación para que dé comienzo la solidificación. Esta anomalía ocurre principalmente en fases iniciales de la solidificación, de forma que como este estudio se centra en la zona latente sin abordar específicamente el comienzo de la solidificación, el subenfriamiento es despreciado.

(c) Otra propiedad de las mezclas homogéneas es que el cambio de fase ocurre en un pequeño rango de temperaturas en vez de a una única temperatura, como es el caso de sustancias puras [22]. Este es el caso del PCM, que es agravado por el hecho de que este rango de temperaturas depende de si se trata de un proceso de congelación o de fusión, con lo que se genera un ciclo de histéresis entre carga y descarga. Este efecto es despreciado en este estudio.

\subsection{ESFERAS DE PCM}

Durante el proceso de carga de frío, se asume que un único frente de congelación avanza hacia el interior de la esfera PCM. La Figura 4(a) muestra el modelo de una esfera PCM durante la carga. 


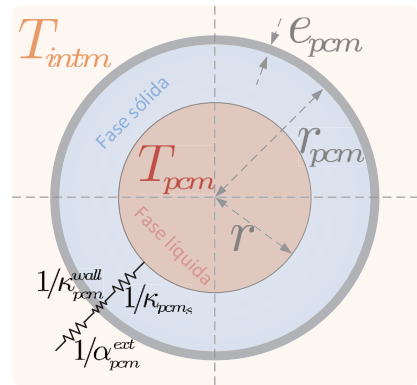

(a) Diagrama esquemático de la esfera de PCM durante la carga de frío

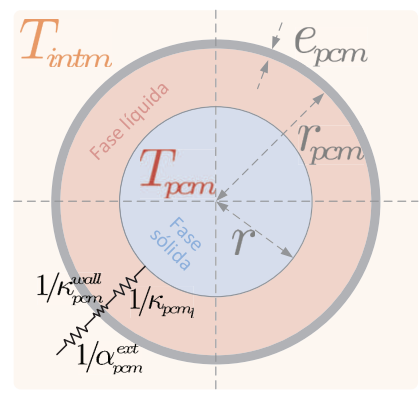

(b) Diagrama esquemático de la esfera de PCM durante la descarga de frío

Figura 4: Modelo de la esfera de PCM en carga y descarga

Se define $r(t)$ como el radio instantáneo del límite esférico entre la fase sólida y líquida dentro de la cápsula. El núcleo fundido decrece mientras la corteza esférica exterior congelada va creciendo hacia el interior de la cápsula. Se considera que el sistema se encuentra completamente descargado antes de la fase de carga, por tanto $r(t=0)=r_{p o m} \mathrm{y}$ $h_{\text {pom }}(t=0)=h_{\text {pom }}^{\text {lat }}$, y que la fase de carga finaliza cuando $r(t)$ se anula.

Por otro lado, durante el proceso de descarga de frío, se asume que un único frente de fusión avanza hacia el interior de la esfera PCM. La Figura 4(b) muestra el modelo de la esfera PCM durante la descarga. En este caso el núcleo sólido decrece mientras la corteza esférica exterior fundida va creciendo hacia el interior de la cápsula. Se considera que el sistema se encuentra completamente cargado antes de la fase de descarga, por tanto $r(t=0)=r_{p c m}$ y $h_{p c m}(t=0)=h_{p c m}^{\text {lat- }}$, y que la fase de descarga finaliza de nuevo cuando $r(t)$ se anula.

Nótese que en la Figura 4 se utiliza la analogía eléctrica para modelar la transferencia de calor entre el núcleo en zona latente de la esfera PCM y el fluido intermedio que la baña. La evolución de $r(t)$ viene definida por $(1)$, donde $\dot{Q}_{\text {pcm }}(t)$ es la potencia térmica transferida entre el fluido intermedio y la esfera PCM, que se calcula mediante analogía eléctrica utilizando (2). Es importante señalar que $\dot{r}(t)<0$ en carga y descarga.

$$
\begin{gathered}
\dot{Q}_{p c m}(t)=\mp \rho_{p c m} h_{p c m}^{l a t} 4 \pi r(t)^{2} \dot{r}(t) \\
\dot{Q}_{p c m}(t)=\frac{T_{i n t m}(t)-T_{p c m}}{R_{p c m}(t)}= \\
=\frac{T_{p c m}-T_{\text {intm }}(t)}{R_{p c m}^{\text {cond int }}(t)+R_{p c m}^{\text {cond, wall }}+R_{p c m}^{\text {conve ext }}(t)}
\end{gathered}
$$

$T_{p c m}$ es la temperatura del núcleo de la esfera de $\mathrm{PCM}$, de forma que la corteza esférica exterior actúa simplemente como una capa conductora entre el núcleo y el fluido intermedio a temperatura $T_{\text {intm }}(t)$. $R_{\text {pom }}(t)$ es la resistencia térmica global, compuesta por tres términos: conducción a través de la corteza esférica exterior de PCM congelado/fundido $\left(R_{p c m}^{c o n d, i n t}(t)\right)$, conducción a través del recubrimiento polimérico de la esfera de PCM $\left(R_{\text {pom }}^{\text {cond, wall }}\right)$ y convección natural en el exterior de la esfera $\left(R_{p c m}^{\infty o n v, e x t}(t)\right)$.

\subsection{FLUIDO INTERMEDIO}

El fluido intermedio se encuentra contenido en el tanque a presión atmosférica, bañando las cápsulas de PCM y los haces de tubos de refrigerante y fluido secundario. Como se ha comentado anteriormente, debido a su alta conductividad térmica, se supone que su temperatura $T_{\text {intm }}(t)$ es homogénea en todo el tanque. El balance de potencias térmicas que afectan a este fluido viene determinado por $(3)$.

$$
\begin{array}{r}
-\rho_{\text {intm }} V_{\text {intm }} c_{p_{i n t m}} \frac{d T_{\text {intm }}(t)}{d t}= \\
=n_{\text {ref }} \dot{Q}_{\text {ref }}(t)+n_{\text {sec }} \dot{Q}_{s e c}(t)+n_{p c m} \dot{Q}_{p c m}(t)-\dot{Q}_{e n v}(t)
\end{array}
$$

$\dot{Q}_{\text {ref }}(t)$ es la potencia térmica transferida con cada tubo de refrigerante, mientras que $\dot{Q}_{s e c}(t)$ es la potencia térmica transferida con cada tubo de fluido secundario. Por su parte, $\dot{Q}_{e n v}(t)>0$ representa las pérdidas térmicas con el entorno. Durante el proceso de carga, no circula fluido secundario a través del tanque y por tanto $\dot{Q}_{\text {sec }}(t)=0$, de forma que el refrigerante proporciona frío al fluido intermedio $\left(\dot{Q}_{r e f}(t)>0\right)$ mientras que las cápsulas de PCM almacenan dicha energía tomándola del fluido intermedio $\left(\dot{Q}_{p c m}<0\right)$. Durante el proceso de descarga no circula refrigerante y por tanto $\dot{Q}_{\text {ref }}(t)=0$, de forma que las cápsulas de PCM transfieren frío al fluido intermedio $\left(\dot{Q}_{p c m}(t)>0\right)$, y el fluido secundario lo toma del fluido intermedio $\left(\dot{Q}_{\mathrm{sec}}(t)<0\right)$. 


\subsection{REFRIGERANTE Y FLUIDO SECUNDARIO}

Durante la carga, el refrigerante circula a través de sus tubos bañados por el fluido intermedio. Se ha hecho uso del enfoque denominado moving boundary para modelar el comportamiento del refrigerante [15], que entra en el tanque en zona bifásica y puede salir del mismo como vapor sobrecalentado (modo 1), o bien como una mezcla bifásica con mayor título de vapor que a la entrada (modo 2$)$.

La Figura 5 muestra un diagrama de la transferencia de calor dentro del tanque, incluyendo también la transferencia con el fluido secundario. En cuanto al refrigerante, se ha representado el modo 1 , en el cual se ha dividido el tubo en dos zonas de longitud variable, donde el refrigerante es bifásico y vapor sobrecalentado, respectivamente.

Se utiliza el método $\varepsilon-N T U[3,8]$ para el cálculo de las potencias térmicas $\dot{Q}_{r e f}$ y $\dot{Q}_{\text {sec }}$, así como la analogía eléctrica representada en la Figura 5. Nótese que las resistencias térmicas globales en los tubos incluyen la convección forzada del fluido circulante en el interior del tubo, la conducción a través de la pared y la convección libre del fluido intermedio en torno al tubo.

\section{RESULTADOS DE SIMULACIÓN}

A continuación se analizan simulaciones de carga y descarga completas en zona latente del tanque TES. Se ha seleccionado el que debe trabajar con el fluido secundario a $-20^{\circ} \mathrm{C}$, de forma que su temperatura de fusión es $-30^{\circ} \mathrm{C}$. La Figura 6 muestra la evolución dinámica de algunas variables durante un proceso de descarga completa en zona latente, en el que se parte de la esfera de PCM completamente sólida (sistema cargado) y se inyecta un caudal constante de fluido secundario a través de los tubos, en condiciones similares a las utilizadas en el evaporador de la planta experimental de refrigeración. Se observa cómo a medida que $r$ tiende a 0 la potencia intercambiada $\dot{Q}_{p c m}$ disminuye en valor absoluto, debido a que la resistencia térmica de conducción debida a la capa fundida es mayor. Como consecuencia, el fluido intermedio aumenta su temperatura, y la temperatura de salida del fluido secundario cada vez es más cercana a la de entrada, al transferirse menos frío desde el fluido intermedio.
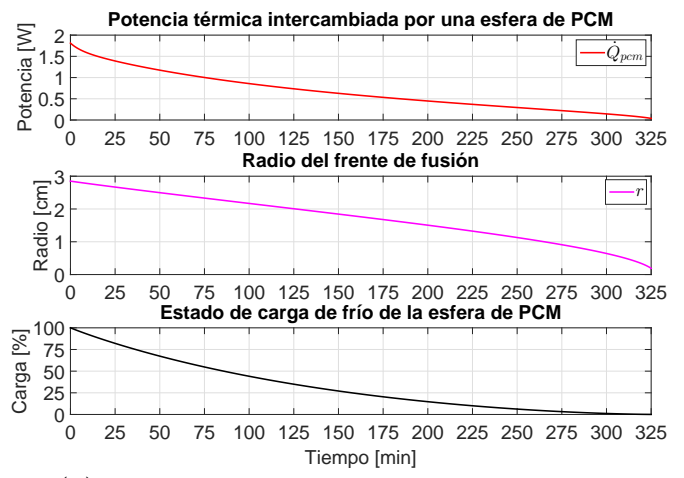

(a) Variables de una esfera de PCM

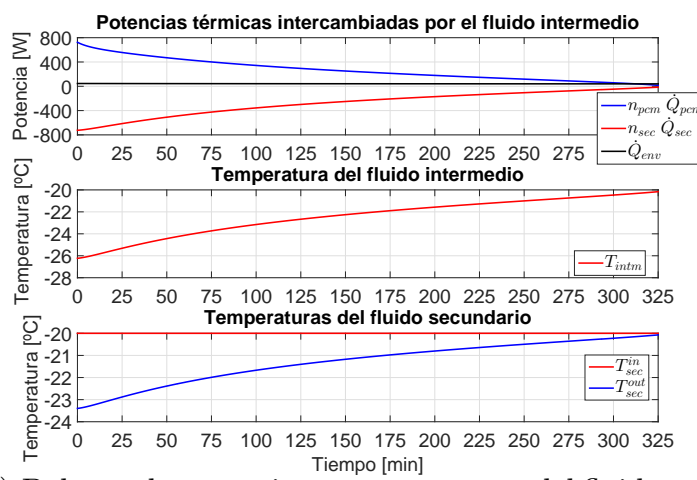

(b) Balance de potencias y temperaturas del fluido intermedio y fluido secundario

Figura 6: Descarga completa en zona latente

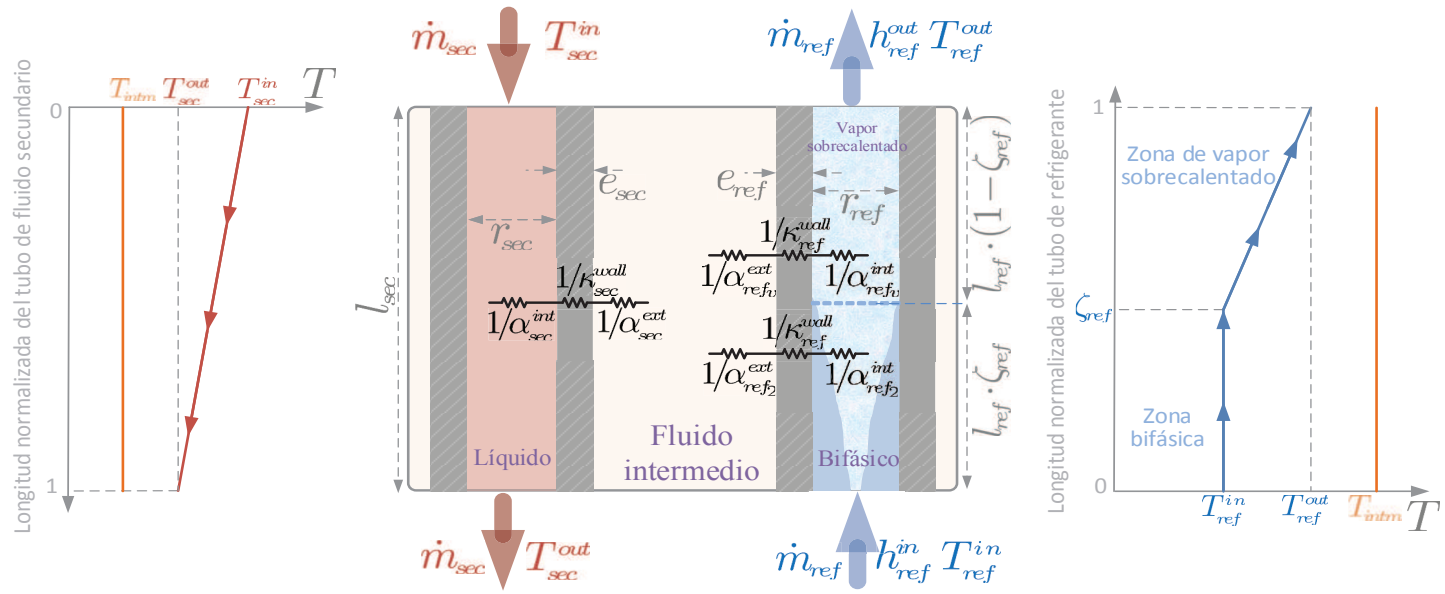

Figura 5: Diagrama de transferencia de calor a lo largo de los tubos de refrigerante y fluido secundario 
La Figura 7 muestra la evolución de algunas variables durante una carga completa en zona latente, en el que se parte de la esfera de PCM completamente fundida (sistema descargado) y se inyecta un caudal constante de refrigerante a través de los tubos, en condiciones entálpicas y de presión propias de la salida de la válvula de expansión correspondiente al evaporador a $-20^{\circ} \mathrm{C}$ de la planta experimental de refrigeración. Nótese cómo $\dot{Q}_{p c m}$ también disminuye en valor absoluto conforme la capa congelada crece. Por tanto, la potencia transferida desde las esferas de PCM al fluido intermedio también disminuye y el refrigerante disminuye su sobrecalentamiento a la salida del tanque TES, de forma que aproximadamente a la mitad de la carga empieza a salir del mismo en estado bifásico.
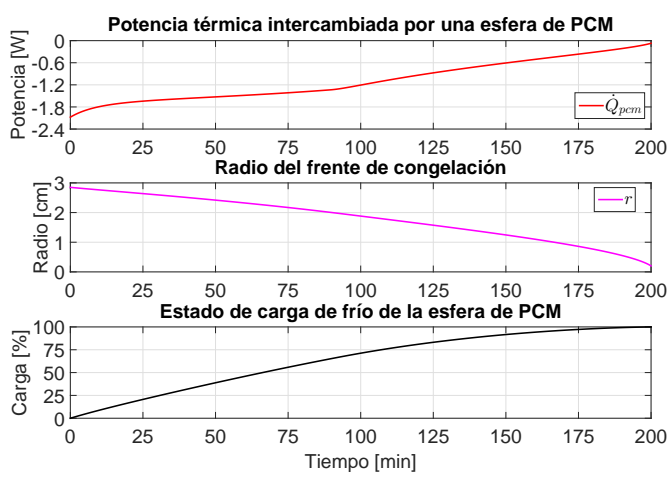

(a) Variables de una esfera de PCM
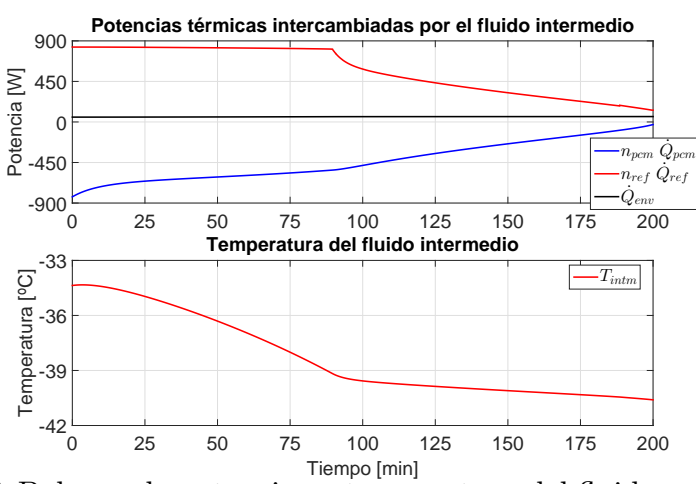

(b) Balance de potencias y temperatura del fluido intermedio
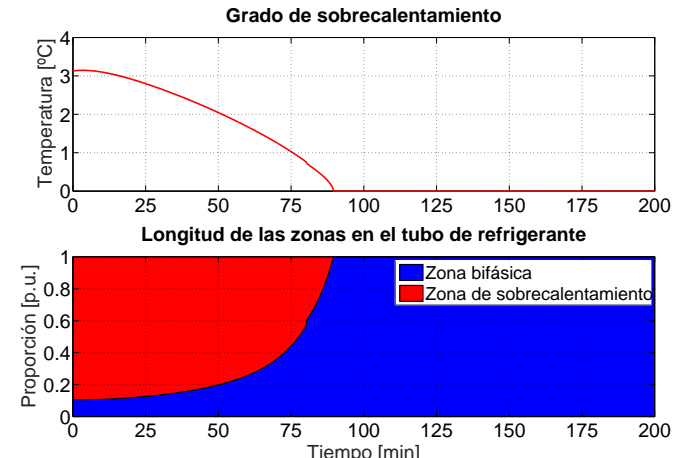

(c) Sobrecalentamiento y distribución de zonas del refrigerante

Figura 7: Carga completa en zona latente

\section{CONCLUSIONES Y TRABAJOS FUTUROS}

Se ha propuesto un modelo dinámico simplificado para un tanque TES basado en PCM, de acuerdo al diseño del mismo para ser acoplado a una planta experimental de refrigeración. Este tanque TES permitirá la gestión del frío generado por la planta según criterios económicos. De acuerdo con las simplificaciones propuestas, se ha desacoplado el modelo en ecuaciones diferenciales para las esferas de PCM y el fluido intermedio que constituye el lecho, de forma que las variables de estado son el radio del frente de fusión/congelación en la esfera y la temperatura del fluido intermedio. Se han presentado resultados de simulaciones de carga y descarga completas, donde se ha observado la influencia del crecimiento de la corteza esférica sólida/fundida en la potencia térmica transferida por la esfera de PCM y por tanto en su velocidad de carga y descarga, así como en las condiciones del refrigerante y el fluido secundario a la salida de los tubos.

Este modelo solo es válido para procesos de carga/descarga completos, ya que si por ejemplo a una carga parcial le sigue una descarga, se crea un nuevo frente de fusión/congelación debido a que el cambio de fase siempre comienza desde la periferia de la esfera. Esto multiplica el número de estados en operaciones parciales, por lo que se plantea como trabajo futuro desarrollar un modelo discretizado que permita estudiar la operación parcial del sistema sin aumentar el número de estados.

\section{Agradecimientos}

Los autores quieren expresar su agradecimiento al Ministerio de Ciencia e Innovación por la financiación de este trabajo, a través de los proyectos DPI2015-70973-R y DPI2016-79444-R.

\section{Referencias}

[1] N. A. M. Amin, M. Belusko, and F. Bruno. An effectiveness-NTU model of a packed bed PCM thermal storage system. Appl. Energy, 134:356-362, 2014.

[2] G. Bejarano, J. A. Alfaya, M. G. Ortega, and F. R. Rubio. Design, automation and control of a two-stage, two-load-demand experimental refrigeration plant. In $23^{\text {rd }}$ Mediterranean Conf. on Control and Autom., Torremolinos (Spain), pages 537-544, 2015.

[3] T. L. Bergman, F. P. Incropera, A. S. Lavine, and D. P. Dewitt. Fundamentals of heat and mass transfer. John Wiley \& Sons, 7th edition, 2011. 
[4] L. O. S. Buzelin, S. C. Amico, J. V. C. Vargas, and J. A. R. Parise. Experimental development of an intelligent refrigeration system. Int. J. Refrig., 28(2):165-175, 2005.

[5] Cristopia Energy Systems. http://cristopia.com/.

[6] I. Dincer. On thermal energy storage systems and applications in buildings. Energy and Build., 34(4):377-388, 2002.

[7] I. Dincer and M. Rosen. Thermal energy storage: systems and applications. John Wiley \& Sons, 2002.

[8] J. P. Holman. Heat transfer. MacGraw-Hill Inc, 8th edition, 2001.

[9] K. A. Jahangeer, A. A. O. Tay, and M. R. Islam. Numerical investigation of transfer coefficients of an evaporatively-cooled condenser. Appl. Therm. Eng., 31(10):1655-1663, 2011.

[10] M. M. MacCracken. Thermal energy storage myths. Energy Eng., 101(4):69-80, 2004.

[11] H. Mehling and L. F. Cabeza. Heat and cold storage with PCM. Springer, 2008.

[12] A. Mosaffa, L. G. Farshi, C. I. Ferreira, and M. Rosen. Advanced exergy analysis of an air conditioning system incorporating thermal energy storage. Energy, 77:945-952, 2014.

[13] A. Mosaffa, C. I. Ferreira, F. Talati, and M. Rosen. Thermal performance of a multiple PCM thermal storage unit for free cooling. Energy Convers. and Manag., 67:1-7, 2013.

[14] E. Oró, A. De Gracia, A. Castell, M. Farid, and L. Cabeza. Review on phase change materials (PCMs) for cold thermal energy storage applications. Appl. Energy, 99:513-533, 2012.

[15] B. P. Rasmussen and A. G. Alleyne. Dynamic modeling and advanced control of air conditioning and refrigeration systems. Technical report, Air Cond. and Refrig. Cent., Coll. of Eng., University of Illinois at UrbanaChampaign., 2006.

[16] B. P. Rasmussen, A. Musser, and A. G. Alleyne. Model-driven system identification of transcritical vapor compression systems. IEEE Trans. Control Syst. Technol., 13:444451, 2005.

[17] A. F. Regin, S. Solanki, and J. Saini. Heat transfer characteristics of thermal energy storage system using PCM capsules: a review. Renew. and Sustain. Energy Rev., 12(9):2438-2458, 2008.

[18] B. Rismanchi, R. Saidur, G. BoroumandJazi, and S. Ahmed. Energy, exergy and environmental analysis of cold thermal energy storage (CTES) systems. Renew. and Sustain. Energy Rev., 16(8):5741-5746, 2012.

[19] P. Schalbart, D. Leducq, and G. Alvarez. Icecream storage energy efficiency with model predictive control of a refrigeration system coupled to a PCM tank. Int. J. of Refrig., 52:140-150, 2015.

[20] S. E. Shafiei and A. Alleyne. Model predictive control of hybrid thermal energy systems in transport refrigeration. Appl. Therm. Eng., $82: 264-280,2015$.

[21] S. E. Shafiei, J. Stoustrup, and H. Rasmussen. Model predictive control for flexible power consumption of large-scale refrigeration systems. In Am. Control Conf. (ACC), 2014, pages 412-417. IEEE, 2014.

[22] W. Streicher, J. Bony, S. Citherlet, A. Heinz, P. Puschnig, H. Schranzhofer, and J. Schultz. Simulation models of PCM storage units. Technical report, Report of IEA Solar Heat. and Cool. Program., 2008.

[23] N. Tay, M. Belusko, and F. Bruno. Experimental investigation of tubes in a phase change thermal energy storage system. Appl. Energy, 90(1):288-297, 2012.

[24] US Energy Information Administration. Residential energy consumption survey (RECS). Technical report, Energy Inf. Adm., Washington D.C, USA, 2009.

[25] F. Wang, G. Maidment, J. Missenden, and R. Tozer. The novel use of phase change materials in refrigeration plant. Part 1: Experimental investigation. Appl. Therm. Eng., 27(17):2893-2901, 2007.

[26] F. Wang, G. Maidment, J. Missenden, and R. Tozer. The novel use of phase change materials in refrigeration plant. Part 2: Dynamic simulation model for the combined system. Appl. Therm. Eng., 27(17):2902-2910, 2007.

[27] F. Wang, G. Maidment, J. Missenden, and R. Tozer. The novel use of phase change materials in refrigeration plant. Part 3: PCM for control and energy savings. Appl. Therm. Eng., 27(17):2911-2918, 2007. 